\title{
Dialysis and Quality of Dialysate in Southeast Asian Developing Countries
}

\author{
Tomotaka Naramura ${ }^{a, b}$ Toru Hyodo ${ }^{a, c}$ Kenichi Kokubo ${ }^{a, d}$ \\ Hirokazu Matsubara ${ }^{a}$ Haruki Wakai ${ }^{a}, c$, e Fumitaka Nakajima ${ }^{a}$ \\ Nobuhisa Shibahara $^{a}$ Kazunari Yoshida ${ }^{a, c}$ Yoshinori Komarua

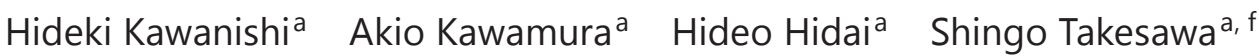 \\ ${ }^{a}$ NGO Ubiquitous Blood Purification International, Yokohama, b Department of Medical Risk \\ and Crisis Management, Faculty of Risk and Crisis Management, Chiba Institute of Science, \\ Choshi, ' Department of Urology, Kitasato University School of Medicine, and d School of \\ Allied Sciences, Kitasato University, Sagamihara, e Shinagawa Garden Clinic, Tokyo, and \\ ${ }^{f}$ Graduate School of Health Science, Kyushu University of Health and Welfare, \\ Nobeoka, Japan
}

\section{Key Words}

Dialysis status - Southeast Asia - Developing countries - Dialysate quality · Endotoxin · Bacterial count

\section{Abstract}

Background: The number of dialysis patients has been increasing in Southeast Asia, but statistical data about these patients and on the quality of dialysates in Southeast Asian dialysis facilities are still imprecise. For this study, dialysis-related statistical data were collected in Southeast Asia. Methods: A survey of the quality of dialysates was carried out at 4 dialysis facilities in Vietnam and Cambodia. The dialysis patient survey included the numbers of dialysis facilities and patients receiving dialysis, a ranking of underlying diseases causing the initiation of dialysis, the number of patients receiving hemodialysis (HD)/on-line hemodiafiltration/continuous ambulatory peritoneal dialysis, the number of HD monitoring devices installed, the cost of each session of dialysis (in USD), the percentage of out-of-pocket payments, and the 1-year survival rates of the dialysis patients (in percent). The dialysate survey covered the endotoxin (ET) level and bacterial count in tap water, in water filtered through a reverse osmosis system and in dialysate. Results: In each of the countries, the most frequent reason for the initiation of dialysis is diabetes mellitus. HD is usually carried out according to the 'reuse' principle. The 1-year survival rates are $70 \%$ in Myanmar and about $90 \%$ in the Philippines and Malaysia. The ET levels in standard dialysates were satisfactory at 2 facilities. The bacterial counts in dialysates were not acceptable at any of the facilities investigated. Conclusion: There is an urgent need to teach medical workers involved in dialysis how to prepare sterile and ET-free dialysates. 
Naramura et al.: Dialysis and Quality of Dialysate in Southeast Asian Developing Countries

\section{Introduction}

The numbers of dialysis patients and facilities in Southeast Asian developing countries have increased substantially, but precise data are unavailable. There have not been any reports about dialysate quality in these countries. In this study, the number of dialysis patients and related statistical data were collected from these countries. The dialysate quality survey was carried out at multiple dialysis facilities in Vietnam and Cambodia.

Study 1 aimed to assess the current status of dialysis practice in Southeast Asian developing countries. Study 2 aimed to investigate the quality of dialysates used at dialysis facilities in Vietnam and Cambodia.

\section{Subjects and Methods}

\section{Study 1}

A survey of the status of dialysis in Southeast Asian countries as of July 1, 2011, was carried out with the support of 9 Japanese dialysis-related companies which have overseas marketing channels. Each company was asked to fill out the survey form on the basis of local information collected by the company. The survey included the numbers of dialysis facilities and patients receiving dialysis in each country, a ranking of underlying diseases causing the initiation of dialysis, the number of patients receiving hemodialysis (HD)/ on-line hemodiafiltration (HDF)/continuous ambulatory peritoneal dialysis (CAPD), the number of HD machines (consoles) installed, the cost of each session of dialysis (in USD), the percentage of out-of-pocket payments, and the 1-year survival rates of the dialysis patients (in percent).

\section{Study 2}

A survey team, including an expert in dialysate purification, was sent for a period from July 31 to August 7, 2011, to investigate the quality of dialysates used at local dialysis facilities, with the cooperation of 3 Vietnamese facilities (facilities A-C) and 1 Cambodian facility (facility D). The survey covered the endotoxin (ET) level [ET units (EU) per liter] and bacterial count [colony-forming units (CFU) per milliliter] in tap water, in water filtered through a reverse osmosis (RO) system and in dialysate. At facilities A, B and C, ET was measured on site. At facility D, samples were collected and combined with a preservative (to minimize ET inactivation), and these samples were carried back to Japan for measurement of ET. The bacterial count was analyzed on the pictures sent via e-mail by a local physician who had photographed the culture medium inoculated with the sample using a digital camera.

The device used for ET measurement was a Toxinometer Mini (Wako Pure Chemical Industries Ltd., Osaka, Japan) in combination with the ET measurement reagent Limulus ES-II Single Test Wako (Wako Pure Chemical Industries). Sampling involved a Contamiless Sampling Port (Medicalseed Co. Ltd., Nobeoka, Japan) and a 1-ml syringe for diabetes (supplied by the local facility; manufacturer unknown). The sample $(0.2 \mathrm{ml})$ was combined with the reagent for measurement. The detectable limit was $0.001 \mathrm{EU} / \mathrm{ml}(=1 \mathrm{EU} / \mathrm{l})$. Measurement was done by the turbidimetric method.

The R2A culture medium used was BBL R2A Agar (Becton, Dickinson and Co., USA). The half-dried sample $(1 \mathrm{ml})$ was applied to the medium, followed by 2 weeks of incubation at room temperature $\left(23-28^{\circ} \mathrm{C}\right)$. The colonies were then counted. 
Table 1. Number of dialysis facilities and patients
Naramura et al.: Dialysis and Quality of Dialysate in Southeast Asian Developing Countries

\begin{tabular}{lrlr}
\hline & Patients, $\mathrm{n}$ & $\begin{array}{l}\text { Percentage of } \\
\text { population }\end{array}$ & Facilities, $\mathrm{n}$ \\
\hline Cambodia & 200 & 0.001 & 10 \\
Myanmar & 600 & 0.001 & 28 \\
Philippines & 10,000 & 0.011 & 270 \\
Vietnam & 14,000 & 0.015 & 130 \\
Malaysia & 23,500 & 0.083 & 600 \\
Thailand & 29,500 & 0.044 & 500 \\
Korea & 45,009 & 0.093 & 614 \\
Taiwan & 63,655 & 0.275 & 552 \\
China & 272,000 & 0.020 & 3,500 \\
Japan & 297,126 & 0.232 & 4,152 \\
\hline
\end{tabular}

Table 2. The three most frequent diseases causing initiation of dialysis

\begin{tabular}{llll}
\hline & 1st & 2nd & 3rd \\
\hline Cambodia & DM & CGN & - \\
Myanmar & HT & DM & CGN \\
Philippines & DM & HT & kidney injury \\
Vietnam & unknown & unknown & unknown \\
Malaysia & DM & unknown & HT \\
Thailand & DM & CGN & unknown \\
Korea & DM & HT & CGN \\
Taiwan & DM & CGN & interstitial nephritis \\
China & CGN & DM & HT \\
Japan & DM & CGN & nephrosclerosis \\
\hline
\end{tabular}

$\mathrm{DM}=$ Diabetes mellitus; $\mathrm{CGN}=$ chronic glomerulonephritis; $\mathrm{HT}=$ hypertension.

\section{Results}

\section{Study 1}

As shown in table 1, the number of dialysis facilities in Asia has increased. In developed Asian countries such as Japan, Taiwan and Korea, the proportion of patients receiving dialysis has reached $0.1-0.2 \%$ of the population. In Cambodia, there were only 2 dialysis facilities as of March 2010 (located in Phnom Penh only), but their number increased to 10 during the subsequent year. In each country, the most frequent cause of renal failure resulting in the initiation of dialysis is diabetes mellitus, as shown in table 2. On-line HDF has been spreading not only in developed but also developing countries. The exact number of patients receiving on-line HDF in Japan is unknown because no survey focusing specifically on on-line HDF has been carried out. In each of the countries, CAPD is not as widespread as HD (tables 3, 4). The cost of each session of dialysis and the percentages of out-of-pocket payments vary among the countries, but the expenses tend to be higher in the developed countries. In the developing countries, such as Cambodia and Vietnam, HD is usually carried out according to the 'reuse' principle. The cost of dialysis is affected by the presence or absence of reuse. The cost of CAPD is higher in the developed countries as the price of the CAPD device/materials is determined by the economic status of the individual countries. The cost of CAPD is quite high in Japan (table 5). The 1-year survival rate of dialysis patients is slightly lower than $90 \%$ in 
Table 3. Numbers of patients on $\mathrm{HD}$, on-line HDF and CAPD
Table 4. Numbers of HD and on-line HDF consoles

Table 5. Cost of dialysis (USD) and percentage of out-of-pocket payments
Naramura et al.: Dialysis and Quality of Dialysate in Southeast Asian Developing Countries

\begin{tabular}{lrlr}
\hline & HD & On-line HDF & CAPD \\
\hline Cambodia & 200 & 0 & 0 \\
Myanmar & 600 & 0 & 0 \\
Philippines & 9,300 & unknown & 700 \\
Vietnam & 10,000 & $5-10$ & 2,000 \\
Malaysia & 21,700 & unknown & 1,800 \\
Thailand & 26,500 & 1,400 & 1,500 \\
Korea & 37,391 & 4,000 & 7,618 \\
Taiwan & 55,825 & 2,200 & 6,110 \\
China & 260,000 & unknown & 12,000 \\
Japan & 297,126 & unknown & 9,728 \\
\hline
\end{tabular}

\begin{tabular}{lcl}
\hline & HD consoles & On-line HDF consoles \\
\hline Cambodia & 75 & 0 \\
Myanmar & 130 & 0 \\
Philippines & 1,870 & 70 \\
Vietnam & 2,000 & 3 \\
Malaysia & 6,500 & unknown \\
Thailand & 5,000 & 250 \\
Korea & 33,900 & 500 \\
Taiwan & 15,040 & 430 \\
China & 30,000 & unknown \\
Japan & 118,135 & unknown \\
\hline
\end{tabular}

\begin{tabular}{lccl}
\hline & $\begin{array}{l}\text { HD } \\
\text { (per session) }\end{array}$ & $\begin{array}{l}\text { CAPD } \\
\text { (monthly) }\end{array}$ & $\begin{array}{l}\text { Percentage of out-of- } \\
\text { pocket payments }\end{array}$ \\
\hline Cambodia & 55 & 570 & 100 \\
Myanmar & 55 & 750 & 100 \\
Philippines & 50 & 240 & 80 \\
Vietnam & 30 & 300 & 0 \\
Malaysia & 50 & unknown & 0 \\
Thailand & 25 & 500 & $0-25$ \\
Korea & 130 & 1,200 & 10 \\
Taiwan & 170 & 2,000 & 0 \\
China & 90 & 800 & $5-30$ \\
Japan & 350 & 5,000 & 0 \\
\hline
\end{tabular}

the developed countries. Data on 1-year survival are unavailable in many countries. The rate is $70 \%$ in Myanmar and about $90 \%$ in the Philippines and Malaysia (table 6). The dialyzer was reused in all the developing countries. Japan, Korea and Taiwan followed a dialyzer single-use principle.

\section{Study 2}

As reference information, the dialysate quality standards prepared by the Japanese Society for Dialysis Therapy are presented below [1]. In RO water and standard dialysate, the 
Table 6. One-year survival rate (\%)
Naramura et al.: Dialysis and Quality of Dialysate in Southeast Asian Developing Countries

\begin{tabular}{lll}
\hline & HD & CAPD \\
\hline Cambodia & unknown & not started \\
Myanmar & 70 & 70 \\
Philippines & 90 & 90 \\
Vietnam & unknown & unknown \\
Malaysia & 89 & 87 \\
Thailand & unknown & unknown \\
Korea & 94 & 94 \\
Taiwan & 87.5 & 89.8 \\
China & unknown & unknown \\
Japan & 87.4 (both HD and CAPD) \\
\hline
\end{tabular}

Table 7. Quality of tap water, RO water and dialysates

\begin{tabular}{lrr}
\hline & ET, EU/l & Bacteria, CFU/ml \\
\hline Facility A & & \\
Tap water & 5,076 & 200 \\
RO water & 741 & 100 \\
Dialysate 1 & 500 \\
Dialysate 2 & & 800 \\
Facility B & 378 & 70 \\
Tap water & 2.9 & 300 \\
RO water & 38 & 150 \\
Dialysate 1 & 49 & 100 \\
Dialysate 2 & & \\
Facility C & 1,468 & 110 \\
Tap water & 94 & 22 \\
RO water & 201 & 600 \\
Dialysate 1 & 395 & 1,000 \\
Dialysate 2 & 2,295 & 1,000 \\
Dialysate 3 & & \\
Facility D & 1,663 & 110 \\
Tap water & 7.3 & 120 \\
RO water 1 & 7.8 & 100 \\
R0 water 2 & 309 & 800 \\
Dialysate 1 & 246 & 800 \\
Dialysate 2 & & \\
\hline
\end{tabular}

${ }^{a}$ At a console with an ET-retentive filter.

ET level should be $<50 \mathrm{EU} / \mathrm{l}$ and the bacterial count $<100 \mathrm{CFU} / \mathrm{ml}$. In ultrapure dialysate injected directly into the human body (e.g. for on-line HDF), the ET level should be $<1 \mathrm{EU} / \mathrm{l}$ (less than the detectable limit) and the bacterial count $<0.1 \mathrm{CFU} / \mathrm{ml}$.

When compared with the standard levels given above, the ET levels in RO water were acceptable at 2 facilities during the present survey. Because no on-line HDF and high-performance dialyzers were used at the facilities investigated, ET levels in dialysis fluids were assessed only on standard dialysis. The ET levels in standard dialysates were satisfactory only at facility B. The bacterial counts in RO water were acceptable only at facility C. The bacterial counts in dialysates were not acceptable at any of the facilities investigated (table 7). 
Naramura et al.: Dialysis and Quality of Dialysate in Southeast Asian Developing Countries

\section{Discussion}

Study 1 revealed that dialysis therapy has been rapidly spreading in Asian countries. As pointed out previously, the numbers of dialysis facilities and patients receiving dialysis seem to be proportional to the gross domestic product. The number of dialysis patients is smaller in poorer countries [2,3]. However, even in countries like Cambodia where this was previously considered unlikely or impossible, dialysis therapy has been spreading steadily. The recent economic growth in Southeast Asian countries has been remarkable. In Vietnam, a national health insurance system is available, with the percentage of out-of-pocket payments being $0 \%$. Study 2 reveals that these developing countries need to acquire considerable know-how about water purification. A low quality of dialysates has many disadvantages for dialysis patients [4-6]. As soon as possible, detailed know-how about how to purify dialysates should be taught to medical workers conducting dialyses by personnel who are specially trained to manage the relevant medical machines and devices in these developing countries. Training courses should be established for clinical engineers working in dialysis units.

\section{References}

1 Kawanishi H, Akiba T, Masakane I, Tomo T, Mineshima M, Kawasaki T, Hirakata H, Akizawa T: Standard on microbiological management of fluids for hemodialysis and related therapies by the Japanese Society for Dialysis Therapy 2008. Ther Apher Dial 2009;13:161-166.

-2 Grassmann A, Gioberge S, Moeller S, Brown G: ESRD patients in 2004: global overview of patient numbers, treatment modalities and associated trends. Nephrol Dial Transplant 2005;20:2587-2593.

3 Hidai H: Inequality of renal replacement therapy in the low-income countries. Nephrol Dial Transplant 2007; 22:663-665.

4 Sitter T, Bergner A, Schiffl H: Dialysate related cytokine induction and response to recombinant human erythropoietin in haemodialysis patients. Nephrol Dial Transplant 2001;15:1207-1211.

-5 Schiffl H, Lang SM, Stratakis D, Fischer R: Effects of ultrapure dialysis fluid on nutritional status and inflammatory parameters. Nephrol Dial Transplant 2001;16:1863-1869.

6 Schiffl H, Lang SM, Fischer R: Ultrapure dialysis fluid slows loss of residual renal function in new dialysis patients. Nephrol Dial Transplant 2002;17:1814-1818. 